\title{
Possibilités et méthodes des politiques locales en direction des Roms migrants : l'importance de la variété d'instruments d'action publique
}

\section{Tommaso Vitale}

tommaso.vitale@sciencespo.fr

Dans toute l'Europe, des camps et baraquements de Roms migrants sont évacués et détruits ${ }^{1}$. En fait «les procédures d'évacuation tiennent aujourd'hui lieu de politique publique en direction des campements illicites » (Cousin, Legros, 2014, p. 1262). Plus en générale, les bidonvilles observés à l'heure actuelle sont issues d'une production des politiques publiques mises en oeuvre : « Les États et les collectivités locales ont amplement contribué à faire des bidonvilles un enjeu structurel pour les métropoles, tantôt oubliés, tantôt considérés comme "problèmes", mais toujours bien réels pour des milliers de citadins » (Aguilera, Vitale, 2015). La question des évacuations est cyclique (Vitale, 2009), réitérée, accompagnée dans la plus parte des cas d'une absence d'alternatives de logement suffisantes (Olivera, 2014), et enfin caractérisée par une violation élémentaire des droits et de la dignité de la personne (Cousin, 2013).

Ce phénomène, aujourd'hui banalisé, renforce les stéréotypes quant à la population rom (Baar, 2011). Si l'une des difficultés de ces destructions de camps réside dans l'absence d'alternative - nous essaierons à la fin de cet article de précisément évoquer ces «alternatives », afin de montrer l'importance d'élargir les instruments d'actions publiques pouvant être utilisés, ainsi que les modalités alternatives afin de les utiliser.

La problématique de cet article est relative à la difficulté des penser et mettre en œuvre des politiques sociales différentiés, par rapport à la multiplicité des situations et compétences des personnes dites Roms dans les villes de l'Europe occidentale (Aguilera, 2014). Le cas des innovations dans les politiques locales italienne sera mobilisé pour développer des réflexions valables aussi en France pour le travail social et les politiques locales en directions de ces groupe.

\section{Une représentation homogénéisant}

Le faiblesse principale de l'action publique locale et nationale en direction de ces groupe est de ne rendre absolument pas compte de la stratification propre des groupes (ces qui par contre est habituelle dans la planification des politiques sociales), ne considérer pas davantage la diversité interne des groupes familiaux ou des groupes familiaux les uns envers les autres. Les compétences de chacun et les individualités ou les moments de vie, ne sont absolument reconnues comme quelque recours que ce soit par les politiques. Les associations mobilisent certes de nombreuses ressources juridiques afin de défendre les droits de chacun, mais la reconnaissance de la capacité de voice des Roms comme interlocuteurs avec lesquels discuter et négocier est très rare, et dépende -entre autre - de l'enjeu pour les "concernés", comme dans n'importe quel dispositif participatif.

Il n'est pas ici de place pour les représentations de singularités, de sorte que les 'traits communs' deviennent une manière de différentier les Roms de la population majoritaire, plutôt que de considérer leur condition dans une ligne de continuité eu égard à la pluralité de leurs conditions de précarité et d'instabilité. Cette représentation largement unifiant est elle même relayée par les médias. Elle est amplifiée par le spectacle de la misère et de la ségrégation et elle finit également par être également endossée par ceux qui, à la base, se déclarent pourtant hostiles aux évacuations : pour beaucoup, également parmi les activistes pour les droits des Roms, ces derniers sont considérés comme un groupe indifférencié, avec une culture homogène et sans différences socioprofessionnelles ou de revenus en leur sein (Vitale, 2009). C'est ainsi que les Roms sont toujours et quoiqu'il arrive considérés comme pauvres et incapables.

1 Cet article développe des principes de méthode esquissés dans Vitale (2015a), qui contienne aussi des données et des evaluation sur la situation des politiques d'intégration voir d'évacuation en France. Des mises à jour quant aux évacuations effectuées dans les villes françaises sont disponibles en ligne à l'adress : http://www.aedh.eu/Recensement-des-evacuations.html. 
Ne pas appréhender la stratification et la pluralité propre aux groupes roms est le produit de politiques publiques démagogiques, alimente des inférences simplistes et crée un fatalisme évident. Beaucoup pensent selon l'expression consacrée que ce sont toujours les mêmes qui depuis trente ans mendient et demandent l'aumône (Belquasmi, 2014). Et aucunes trajectoires d'intégration, de vivre ensemble et de cohabitations satisfaisantes ou de mobilité sociale, ce qui est pourtant souvent le cas, avec par exemple des parcours d'intégration à travers l'entreprenariat ne sont mises en valeur. Aucune confiance et peu de crédits en sont accordés aux politiques sociales de soutien, également à l'égard les familles les plus vulnérables et les plus fragiles, parce que le destin de l'ensemble d'un groupe ethnique semble quoiqu'il arrive d'ores et déjà établi ou préétabli. L'opinion commune finit ainsi par être portée par l'idée que les Roms se plaisent à vivre dans la saleté, ainsi que dans des situations précaires ou que les Roms ne sont guère intéressés par une quelconque qualité de vie et qu'avec eux rien n'est possible. Le tout sans que ceci ne se base sur quelque réalité que ce soit. Ces représentations, rhétoriques classiques de la réaction au sens d'Hirschman (1991), qui font penser que rien est possible, alimentent à leur tour et se nourrissent dans le même temps des pires, des plus anciens et des plus ancrés stéréotypes sur les « Tziganes » : à savoir une réputation de délinquance, et une représentation des enfants ne bénéficiant pas de cette sorte de présomption de bienveillance et d'innocence qui irradie les tous jeunes citadins et leur donne ainsi une sorte d'immunité en public (Sarcinelli, 2012).

\section{La vulnérabilité des groupes Roms}

La situation des groupes tziganes de nouvelle immigration est caractérisée par une forte exclusion sociale d'ensemble; exclusion du marché du travail, de l'éducation, de la santé et des services sociaux. L'analphabétisme et la ségrégation spatiale, l'isolement et le manque de services essentiels sont particulièrement diffus parmi ces groupes, et de manière plus importante encore par rapport à toutes les autres minorités. Pas tous les groupes roms, en tout cas. On parle surtout de groupes de nouvelle immigration, de la Bulgarie et de la Romanie vers l'Europe occidentale. Ces groupes ont rarement recours à l'assistance sanitaire publique en raison, souvent, d'attitudes discriminatoires de la part des structures et du personnel sanitaire, en raison de la ségrégation spatiale dans laquelle ils vivent, mais également en raison d'une conflictualité partielle entre les formes de traitement sanitaire et les attitudes les plus diffuses de soin à la personne d'un coté et, de l'autre, la conception de la pudeur parmi les groupes tziganes. Ceci étant renforcé par l'absence de communication entre communauté tzigane et service sanitaire public, mais aussi par l'absence d'information réciproque. Nous parlons ici, comme dans le cas de l'école, du conflit et de la réciproque étrangeté, entendue comme le fait d'être étranger l'un à l'autre, entre une différence culturelle et un système universaliste. Deux pôles entre lesquels la politique et les institutions sont appelées à créer des formes de médiation et de conciliation (Brüggemann, 2012).

Le manque de formation et de qualification professionnelles, associé à des capacités ou qualifications professionnelles traditionnelles requises de manière toujours plus rare par le marché du travail, le tout en plus, et de nouveau, associé à l'isolement spatial dans lequel les groupes les plus marginaux vivent, sont autant d'éléments constituant une barrière à l'entrée des Roms et des Sintis dans le marché du travail. Les difficultés proviennent également du status légal fort complexe de ces groupes et des discriminations, directes ou indirectes, basées sur les préjugés de leur inclination «naturelle» limitée pour le travail et pour le respect des règles (Vitale, et al., 2009).

L'exclusion sociale frappant les Roms et les Sintis se présente donc tel un phénomène dû à divers facteurs de causalité interconnectés et se combinant entre eux en un processus causal cumulatif à l'intérieur d'un processus lui même et par ailleurs multidimensionnel et intergénérationnel. La ségrégation spatiale, par exemple, a un impact direct sur la santé et sur l'accès aux services, ce au même titre que la faible scolarisation a des effets négatifs sur la capacité à s'intégrer dans le monde du travail, ce qui à son tour complexifie la construction de parcours, afin de précisément sortir de la ségrégation spatiale des camps. Chaque facteur est non seulement connecté avec les autres, mais il a des effets rétroactifs et renforçant sur les autres. Mais si l'on devait identifier un facteur situé au centre de toutes les relations causales, la ségrégation spatiale serait incontestablement celui-ci, élément central de codétermination de toutes les autres formes d'exclusion et de privation. 


\section{Création et mise en place de politiques à l'endroit des groupes tziganes : questions méthodologiques et de design.}

Une fois données ces caractéristiques propres aux phénomènes d'exclusion sociale et de privation des Roms et des Sintis, les recherches conduites au niveau européen conduisent elles à identifier un ensemble très précis de caractéristiques des politiques publiques, ayant de raisonnables possibilités de rejoindre des objectifs de réduction de l'exclusion et, à terme, d'intégration plus large :

a) Variété des instruments de politique publique et intégration des instruments, des structures et des acteurs mettant en œuvre les politiques.

b) Mise en place de processus et de mécanismes, à la fois stabilisés et continus dans le temps, mais modifiables sur la base de l'expérience et des résultats de traduction entre la communauté tzigane et la société majoritaire. Par traduction, nous entendons dans ce cas connaissance réciproque de la culture, des codes et des processus guidant les attitudes respectives et les comportements sociaux.

c) Mise en place et stabilisation des dispositifs institutionnels en mesure de construire et de régler des sphères d'échange, de discussion, de prise de décision et de coordination des politiques entre les différentes institutions et entre institutions, la communauté tzigane et les acteurs de la société civile.

d) Garantir aux politiques une continuité dans le temps, ainsi que les possibilités de vérification nécessaires de celles-ci en fonction de leur capacité progressive d'adaptation quant aux objectifs à rejoindre.

e) Rôle de protagonistes directs de la part des acteurs politiques, des établissements locaux dans la mise en place et la réalisation des politiques et dans la médiation conflictuelle.

f) Intervenir activement dans la déconstruction et l'élimination des deux principaux facteurs structurels de l'exclusion des Roms et des Manouches: la ségrégation spatiale (Legros, Vitale, 2011) et la diffusion des préjugés (Mayer, et al., 2015).

La plus parte de ces design principles sont bien connus et valables dans les politiques sociales. Le premier point (la variété d'instruments), par contre mérite d'être analysé avec attention.

\section{La variété d'instruments comme principe de design}

A partir des années '80, si on prends l'Italie comme exemple, le modèle dominant des politiques envers les Roms et les Sintis a été celui du binôme camp «nomades»/évacuation. Les autres formes d'intervention ont été soit annulées en fonction de ce modèle soit reconduites à ce dernier et donc, en grande partie dans les faits, rendues totalement vaines. Ceci n'est pourtant pas l'unique modèle d'intervention possible mis en place. Car des formes de multiplication des instruments d'intervention de la part des gouvernements locaux sont en effet et toutefois en train d'apparaître. Et une modification des instruments mis en œuvre par les établissements locaux est en ce sens également importante qu'elle a des implications constitutives sur les acteurs impliqués (Halpern, Lascoumes, Le Galès, 2014). Les capacités des acteurs sociaux dépendent en effet fortement des instruments des politiques publiques utilisées et des espaces d'action qu'ils ouvrent.

Le caractère univoque des solutions de logement en camps «nomades» (ou dans les villages d'insertion en France, voir Legros, 2010 ; Bessone, et al., 2013) peut être dépassé par une série hétérogène de solutions en matière de logement :

- $\quad$ logements publics ;

- logements privés avec des activités de soutien aux membres des communautés roms pour ce qui concerne l'accès au crédit et la réputation des familles (afin d'affronter et de dépasser les préjugés que la cohabitation avec des familles roms et sintis impliquent souvent);

- $\quad$ auto-construction sur des terrains de propriété des familles avec le soutien des coopératives ;

- $\quad$ prédisposition de zones équipées, en termes résidentiels, pour des groupes familiaux étendus, lesquels gèrent ces zones en étant soit propriétaires soit locataires ; 
- $\quad$ programmes de logements publics, à travers des appels d'offres publics dirigés également mais pas seulement vers les familles tziganes, afin de ne pas davantage favoriser les préjugés sur les «privilèges» des Roms que de se perdre dans des schémas différentialistes ;

- $\quad$ des aires de stationnement équipées pour les groupes itinérants, par exemple les forains.

Les formules de logement adoptées par les groupes roms sont par ailleurs les plus disparates les unes des autres. En d'autres termes, la question du logement est problématique non seulement en ce qui concerne les conditions d'indigence de certains individus, mais également par l'hétérogénéité des demandes formulées par les différents groupes. Une hétérogénéité qui demande de bonnes capacités de programmation des autorités locales. Chacune des formes de logement existantes présente en effet des problématiques spécifiques et nécessite en conséquence une approche adéquate pour chacune d'entre elles.

La pluralité des interventions ne concerne pas seulement les politiques de logement. Pour ce qui concerne la scolarisation, certains parcours ont également été expérimentés : ils prévoient la promotion de la culture rom à l'intérieur des classes; le soutien éducatif (concernant la participation à des activités collectives, des activités sportives, mais également des laboratoires créatifs) et didactique (directement lié à l'apprentissage et à la réussite scolaire) à l'intérieur des classes, mais aussi, l'après-midi, à l'intérieur même des communautés ; ils prévoient en ce sens des programmes dirigés pas seulement vers les mineurs, mais vers les familles dans leur ensemble, afin d'agir sur les obstacles culturels présents entre les adultes roms et manouches autour de la participation des mineurs et des femmes aux activités scolaires (Bruggeman, 2011); sorties culturelles avec les mineurs roms et sintis afin qu'ils connaissent, et d'une certaine manière, qu'ils commencent à s'approprier les lieux qui entourent les espaces qu'ils habitent; des systèmes de récompenses (des " prix ») pour les mineurs, liées à leur réussite scolaire ; consultation des communautés dans leur ensemble avant de commencer des projets d'insertion scolaire; réalisation de spectacles artistiques, spectacles de théâtre, d'événements culturels, afin de rapprocher le monde de l'école et la communauté rom et sinti ; ils prévoient enfin la présence de médiateurs spécifiquement formés, et ce, de manière approfondie, dans le but spécifique d'intervenir dans les rapports mineurs-communauté-école. Comme dans les autres domaines d'intervention, il était de fait important que les projets suivent ainsi des parcours à la fois individualisés et orientés vers les groupes familiaux singuliers (Vitale, Cousin, 2011).

Pour ce qui concerne le travail, des politiques ont été expérimentées, orientées vers l'accroissement des opportunités et (donc) des capacités des individus et des familles, en termes de développement du capital social et de développement intrinsèque des communautés, le tout en valorisant les ressources, les réseaux et les capacités d'auto-organisation des Roms et des Sintis selon un modèle répondant à l'«active welfare approach» tenant à la fois compte des facteurs directs (préjugés, isolement, manque de formation et de qualification professionnelles), et des facteurs indirects (logement, école, accès aux services sociaux, liens avec la société majoritaire), d'exclusion du monde du travail. Une partie importante des projets d'insertion professionnelle prévoit de plus une série d'activités propédeutiques : compréhension de la part des Roms et des Sintis du contexte urbain d'ensemble dans lequel ils vivent et à agissent ; développement des attitudes à interagir avec la société les entourant, ainsi qu'avec les institutions ; développement enfin de la capacité de compréhension et d'interaction avec le marché du travail.

A des fins d'insertion active dans la réalité professionnelle, des conventions avec des entreprises et des coopératives ont été expérimentées dans le cadre de cours de formation, mais aussi des formes de médiation des acteurs publics et des représentants du troisième secteur, entre les Roms et Sintis et les entreprises. Mais un des points faibles de ces projets d'insertion professionnelle se situe précisément dans la difficulté de trouver et de stimuler une disponibilité de la part des acteurs privés, ce malgré la médiation d'autorités ou d'établissements locaux et d'associations, afin d'offrir des opportunités professionnelles. Comme dans le cadre des politiques de logement, la médiation, même non suffisante, reste toutefois la condition nécessaire afin d'atteindre, en termes de capital, des niveaux nécessaires de réputation et de confiance en mesure d'éroder auprès des privés, les préjugés attachés à la capacité de travail des Roms, des Manouches et des Sintis. Dans de tels cas, il est procédé à la 
mise en place de réseaux entre les institutions publiques, les acteurs du troisième secteur, les privés, les «tsiganes ». Ceci nécessitant, afin de donner des résultats, des financements organisationnels stables et continus. A travers le soutien scolaire, en particulier, un accent particulier a été mis sur des tentatives de développer les capacités spécifiques et les vocations des membres des communautés, et ce notamment à travers des projets d'entrepreneuriat.

Bien sur cet ensemble de politiques se doit d'être intégré. Mais les problèmes de coordination et fragmentation des politiques sociales ne sont pas une spécificité ni une nouveauté, ils sont consubstantiels aux politiques sociales. Ce que on veut souligner ici est que la variété et la coordination des instruments obligent en effet à contraster la réification de cet univers hétérogène de minorités en une communauté administrative unique vouée à un instrument d'intervention unique. La variété et la coordination des instruments impliquent de dépasser de manière positive à la fois le traitement différentiel et les politiques spécialisées (Legros, Olivera, 2014), exclusivement vouées aux communautés tziganes. Politiques spécialisés qui sont en réalité dis-fonctionnelles, au-delà des intentions mêmes de ses promoteurs, à une conciliation entre la spécificité d'une culture et d'une condition sociale d'un côté et les critères universalistes d'accès et de jouissance des services publics de l'autre, contribuant de surcroît à renforcer encore les stéréotypes d'assistances à l'égard des Roms (Stewart, 2013).

De plus, la variété des instruments favorise la prédisposition d'interventions inter scalaires concernant autant les communautés dans leur ensemble - dont la participation est absolument nécessaire, surtout en phase de mise en projet, mais ceci vaut également en cas de conflit avec la population majoritaire ; dans ce type de cas, la présence des acteurs hostiles à la présence tzigane est elle aussi nécessaire au même titre que la médiation des exigences et des besoins qu'ils expriment - que des familles et des individus envisagés de manière singulière. Les interventions de politique publique doivent en effet s'accorder à tous les niveaux de l'expérience sociale des Roms, en partant, tout au long des projets, du noyau le plus large (la communauté) pour progressivement arriver à des parcours quasiindividuels. Dans ce cadre le fait que, comme justement Céline Bergeron nous rappelle, il y a « un déplacement des pratiques de certaines familles Roms qui s'inscrivent, dans un premier temps, dans la migration puis s'en écartent peu à peu pour s'ancrer dans un mode de vie circulant, le plus souvent à l'intérieur même des frontières françaises », en principe n'est pas un élément de difficulté ou une défis laborieux pour les politiques publiques, mais au contraire un levier en direction d'une action plus attentive aux trajectoires individuelles, en continuité avec les développements plus récents et innovantes des politiques d'aide sociale (Vitale, Membretti, 2013 ; voir aussi Duvoux, 2012).

La variété et la coordination des instruments stimulent enfin les différents secteurs de l'administration à prédisposer ces dispositifs de coordination institutionnelle, auxquels nous avons fait référence à plusieurs reprises ici. Dispositifs à l'intérieur desquels les différents secteurs de l'autorité locale concernée se coordonnent entre eux et mettent en communication ou en relation ladite autorité avec d'autres institutions, avec les médiateurs, ainsi qu'avec les destinataires de l'action publique. Ces dispositifs, et les acteurs qui en font partie, peuvent jouer un rôle fondamental dans la traduction réciproque des mondes culturels respectifs, des pratiques sociales et organisationnelles entre communautés tziganes et société majoritaire, mais aussi dans la médiation de situations conflictuelles.

\section{7. (Fausses) Controverses en vue de la programmation d'interventions sociales}

Une partie du débat publique concertante les politiques pour les Rom et les Sinti et, plus encore, des acteurs du système politique et médiatique dépeignent ces politiques tel un champ caractérisé par un système de dichotomie : soutien à la scolarisation diffuse ou scolarisation de l'élite; migrants ou immigrés ; intervention pour les Roms ou intervention des Roms ; financement à projet ou formation sur des programmations ou des mises en place de consensus; sécurité ou politiques sociales ; interlocution avec les communes ou avec les régions ; appels d'offre pour les Roms ou quotas pour les Roms à l'intérieur d'appels d'offre existants.

Les considérations jusqu'alors formulées nous permettent d'affirmer qu'il s'agit là des fausses dichotomies. Les projets d'insertion scolaire qui se sont révélées majoritairement efficaces se posent 
comme objectifs une scolarisation diffuse. Mais une action spécifiquement ciblée sur les élites des communautés, pouvant par exemple fonctionner et garantir la légitimation des projets auprès de la communauté peut être fonctionnelle au fait de rejoindre ou d'atteindre lesdits objectifs. Le plus important se situe alors et quoi qu'il arrive dans le fait que les projets ne visent pas à créer des tensions entre les mineurs et leurs parents, mais soient au contraire conjointement dirigés vers les deux parties. Les expériences «informelles» de formation professionnelle identifiant de petites niches productives et favorisant la formation et l'insertion professionnelles, avec comme partie prenante de petites entreprises familiales, ont par ailleurs un très haut niveau d'effectivité. Pourquoi ? Trois raisons à cela : (1) Elles impliquent le groupe familial dans son ensemble et non pas de jeunes individus envisagés de manière uniquement singulière. Ces expériences tirent donc également leur force des liens familiaux, plutôt que de créer des tensions à l'intérieur des familles ; (2) Elles sont en mesure d'impliquer de petites et moyennes entreprises ancrées territorialement en produisant une formation hautement spécifique et professionnalisante (avec les limites de l'absence de certification cependant) ; (3) elles sont construites dans l'interlocution et dans la discussion directe avec les Roms eux-mêmes et non pas autour d'une table sur la base de représentations stéréotypées de la culture rom et de son caractère de dégradation supposé (Vitale, 2010).

$\mathrm{Au}$ regard de la dichotomie migrants/immigrés nous avons pu observer la radicale hétérogénéité de la condition juridique et du status des Roms et des Sintis. Certaines situations - de privation, de marginalité, de criminalité et de micro-criminalité - sont spécifiquement liées à des groupes d'immigration récente et donc à la condition ou à l'ensemble des problématiques liées à la condition des migrants (Naçu, 2010), et souvent est un produit institutionnel, effet d'un processus d'" illégalisation » des plus démunis (Doytcheva, 2015). Mais il existe également et cependant ici une forte composante concernant des Roms immigrés de seconde et de troisième génération dont la condition elle, n'est pas comparable à la précédente. Il est donc important de discerner, au cas par cas, dans la mise en place des différents projets d'intervention, si et dans quelle mesure spécifique, les situations sont liées à une condition d'immigration récente, ou relève au contraire de facteurs plus sédimentés dans le temps, et quelle est donc la composition des communautés par rapport à ces variables.

Pour ce qui concerne la dichotomie interventions des/pour les Roms, nous avons vu que l'on ne donne guère une chose sans l'autre : chaque intervention efficace pour les Roms est une intervention avec les Roms, et ce, dès la phase de mise en projet (Vitale, Boschetti, 2011). Il s'agit aussi de reconnaître l'insertion existante des activités économiques de service des Roms en complémentarité et avec intérêts convergents (par exemple autour de l'approvisionnement du marché des métaux recyclés) mais, bien plus fréquemment, en compétition avec d'autres acteurs et intérets. Martin Olivera, par exemple, nous rappelle des formes de compétition autour de «l'exploitation des espaces vacants et des goubelles, accès à l'eau et, plus généralement, usage de l'espace public géré par ces sociétés et leurs filiales etc » $(2015$, p. 511). Une intervention efficace donc n'est pas seule planifier avec les Roms, mais aussi elle prends en considération les acteurs avec les quelles ils sont en relation de coopération et de compétition.

De la même manière, le financement des projets avec des objectifs ciblés d'un coté, et la programmation sur le long terme de l'autre, qui prévoit d'importants investissements dans la formation des personnels spécialisés, mais aussi un long et difficile travail de mise en place et de création d'un consensus au sein de la société locale, ne doivent pas - ou ne devraient pas - être posés comme des alternatives s'excluant l'une de l'autre. Chaque projet financé puise son efficacité dans le fait de constituer une partie, un moment, du travail de programmation et de recherche d'un consensus à moyen terme. Un consensus, d'autre part, à construire de manière incrémentale impliquant activement d'un coté les différents segments de la société locale directement ou indirectement intéressés à la réalisation des politiques pour les Roms et les Sintis (également les plus hostiles), et impliquant de l'autre la nécessité de fournir à la même société locale des preuves de réalité leur permettant d'observer de manière pratique des objectifs atteints, même de manière partielle ; un consensus, enfin, nécessaire afin d'aller dans le sens de l'intégration et de la réduction de la marginalité et de la privation de ces minorités. 
La corrélation entre sécurité et politiques sociales est ici évidente. L'efficacité de ces dernières rétroagit positivement sur la première et constitue une preuve de réalité permettant d'élargir le consensus sur les politiques d'intégration. Ceci ne signifie pas pour autant négliger des difficultés spécifiques liées à des problèmes de sécurité, de criminalité et de micro-criminalité à l'intérieur des communautés. Il est en effet admis que - comme dans toutes les situations sociales caractérisées par un bas niveau d'intégration, de pauvreté, de marginalité et de précarité des conditions de vie - les situations de criminalité sont bien présentes à l'intérieur de certains groupes roms. Ces problèmes sont bien entendu à prendre en considération et à affronter de manière conjointe avec les institutions publiques, avec les instruments ordinaires (et non pas avec des instruments d'urgence ou extraordinaires) des politiques de sécurité et de droit pénal, mais des problèmes qui se doivent dans le même temps d'être affrontés, avec une volonté véritable d'agir sur l'intégration et la réduction de la marginalité à travers les politiques sociales.

Pour ce qui concerne les deux dernières fausses dichotomies que nous indiquions, nous avons déjà observé en quoi les projets et les politiques se doivent d'impliquer les institutions dans une dimension pensée à différents niveaux, impliquant autant le niveau municipal que le niveau départemental et, si possible, le niveau régional. A l'intérieur des municipalités, un élément central réside par ailleurs dans l'attribution des responsabilités à des structures administratives spécifiques - conseillers et services municipaux - en mesure de garantir aux politiques et aux projets une continuité dans le temps ; laquelle continuité se devant de pouvoir faire face aux fluctuations que le cycle politique administratif de cinq ans lui impose et qui puisse donc leur garantir une pérennité au delà des contingences politiques. Il est toujours préférable de ne pas alimenter ni des politiques ni des comportements culturels de type différentialiste et des appels d'offre publics spécifiquement adressés aux Roms. Et de fait, les politiques publiques qui ce sont à ce jour révélées être les plus efficaces sont des politiques dans lesquelles des quotas leur étaient réservés à l'intérieur d'appels d'offres dirigés eux vers une population plus large. Bien évidemment toujours en combinaison avec un mélange de critères de méthode pour construire la programmation des interventions : alternatives résidentielles réelles pour tous les sujets, sans écrémages ; stabilité du budget; négociation directe avec les familles ; budget assigné sur une base individuelle (Vitale, 2015b, pp. 64-5); un plan stratégique (avec des objectifs mesurables et vérifiables), et un bureau chargé de suivre la mise en œuvre et d'établir un suivi quotidien des résultats ; un dialogue continu avec la ville.

\section{Construction du consensus, volonté politique et formes de médiation}

Que peut-on à ce point ajouter, sinon que les instruments des politiques publiques demeurent structurellement rétives au changement, caractérisées par une certaine inertie et capables d'influencer à la fois la routine et les choix. Car dans le même temps en effet, c'est bien la politique qui fait la différence. Et ce qui fait la différence, ce ne sont pas seulement les politiques choisies par les décideurs politiques (conseillers et maires), mais également par les oppositions et les partis politiques dans leur ensemble dans leur rapport avec les représentants de structures organisées de la société civiles et des médias.

Ce qui en revanche est pour le moins clair est que la présence de groupes tziganes donnent toujours lieu à conflits : surtout avec les groupes de nouvelle génération, mais pas seulement. Et dans chaque contexte local, les acteurs politiques profitent de ces conflits afin de tenter d'agglomérer le plus de consensus possible : et en ce sens, beaucoup jouent le jeu de l'aggravation du conflit en polarisant les positions de chaque camp.

La polarisation peut être définie, en termes d'issue, comme un échec dans la production de dispositifs capables de stabiliser et d'établir des compromis entre les parties. Ou, en d'autres termes, la polarisation peut être ainsi entendue comme le résultat de processus actifs de destruction de dispositifs institutionnels capables de stabiliser et d'établir des compromis entre les parties. Parmi les grands sociologues, c'est d'abord Simmel (1908) qui attira l'attention sur le fait qu'il ne faille pas seulement s'arrêter à l'issue du conflit, qui gagne, qui perd, que gagne l'un et que perd l'autre, et encore moins à seulement analyser le point d'équilibre portant en lui les contrastes. L'auteur souligne plutôt en quoi la résolution d'un conflit est un processus, un processus de médiation dans la dynamique du conflit. 
La production d'une reconnaissance mutuelle entre les parties en conflit est le résultat de la présence de formes de médiation. En d'autres termes, les conflits quant à la présence des groupes roms et sintis constituent un défi posé aux capacités de médiation institutionnelle pas seulement sur le terrain de l'efficacité, mais également sur celui de la qualité des processus à travers lesquels les matières sociales sont dénommées et identifiées par rapport à des thématiques et des problématiques, par rapport à des règles et à des standards.

Y compris dans des situations tendues, contradictoires, très dures, les cas que nous avons eu l'occasion d'analyser en Italie (Vitale, Caruso, 2009) mettent malgré tout toujours en lumière des aspects négociables sur lesquels les décideurs politiques se sont à la base appuyés, afin d'impliquer toutes les parties en conflit dans un processus politique commun. Les cas de Modène, Mantoue, Buccinasco et Padoue ont par exemple montré le caractère relevant d'opérations de localisation effectuées de manière non dissimulées, garantissant au contraire une information préventive entière, sans se soustraire, mais en s'impliquant au contraire dans le conflit en informant et en discutant par exemple dans les quartiers dans lesquels on entendait promouvoir une implantation pour des Roms ou pour des Sintis. Le tout en se donnant à la fois du temps et des rythmes ramassés. Et trois facteurs font la différence ici : la volonté politique et la cohésion de la coalition de gouvernement quant aux choix effectués en est une, bien sûr. Toutefois, la volonté politique ne suffit pas en ce sens que la qualité du tissus administratif qui la met en œuvre est absolument crucial comme véritable moteur en mesure de favoriser la médiation des conflits. Le style de communication adoptée, enfin, est fondamental en termes de résolution : il s'agit en effet de prendre également au sérieux les revendications de ceux qui protestent, de les écouter, de discuter avec eux dans le respect de tous afin de favoriser une élaboration supplémentaire quant aux argumentations formulées et aux craintes sous-jacentes.

Il est bien entendu possible que les institutions médiatrices d'un conflit, contribuent parfois elles mêmes à la polarisation de ce dernier, comme cela s'est en partie produit à Venise lors d'une première phase de médiation où la majorité, toutefois, s'est révélée en mesure de revoir ses propres modalités d'action, tout en maintenant ses objectifs et sa capacité d'avancer dans la direction qu'elle s'était fixée (Mantovan, 2011).

En quoi consiste les médiations dont nous avons parlé jusqu'alors ? Nous pouvons en l'occurrence repartir ici de cas où les municipalités ont été en mesure de développer des politiques de promotion des capacités des groupes tziganes, en créant un consensus autour de leurs propres choix et en différenciant les formes de médiation des conflits. Nous pouvons à ce titre nous référer ici à une typologie tripartite formulée par Peter Berger (1998), la précisant ici afin de servir notre exposé.

Et les cas de Bologne, de Pise, de Venise, de Rovereto et de Trente présentent un certain nombre de traits communs dans la médiation des conflits ; selon des modalités que l'on pourrait en l'occurrence qualifier ici de médiation impérative. Il s'agit là d'une forme d'arbitrage selon laquelle l'issue de la médiation relève de l'autorité et de la responsabilité du médiateur et aura par la suite un effet contraignant pour l'ensemble des parties. L'autorité locale, après avoir recueilli les informations nécessaires et après avoir écouté les différentes parties, évalue et 'pèse' les compromis et conclue ainsi les dynamiques de la médiation en imposant une issue légitime à laquelle les parties doivent se conformer. Les limites de ce type de médiation résident dans le fait que l'ordre normatif des parties opposées ne se modifie pas nécessairement dans la brève période du processus considéré. Le choix quant à lui ne crée pas de consensus. C'est la raison pour laquelle cette médiation ne semble pas pouvoir créer les conditions nécessaires en mesure de dissiper les tensions. Et c'est également pour cette raison, probablement, que dans l'ensemble des cas analysés, aucun d'entre eux n'est à caractériser à travers une médiation impérative exclusive.

Dans les cas de Buccinasco, de Lyon, de Padoue et de Rovereto, le processus de médiation se distingue par son caractère non coercitif; processus dans lequel les parties cherchent des solutions de compromis en s'appuyant sur la présence d'un tiers. Les organisation du troisième secteur jouent à ce point un rôle crucial, ce au même titre que les organisations publiques mettant à disposition cadres et ressources (en premier des ressources procédurales), afin que les parties traduisent des valeurs en raisons, et surtout, des normes en intérêts, ce en acceptant une référence normative commune (mais non ultime, règles afin de résoudre un conflit sur lesquelles insistait beaucoup Simmel). 
En ce sens, la possibilité de traduction d'intérêts particuliers en un intérêt collectif se base sur des règles partagées et reconnues : et c'est en ce sens que nous pouvons parler ici de médiation pragmatique. C'est une voie possible à parcourir, aussi bien dans les situations politiquement les plus corrompues, et la aussi où les niveaux de ségrégation sont plus hauts (Vitale, 2015b).

D'autre cas, enfin, même à travers le recours à différentes modalités de médiation, permettent d'identifier assez clairement un troisième idéal type. Pensons ici aux cas de Settimo Torinese, de Mantoue, de Modène, et aussi, sous de nombreux aspects, également à ceux de Pise et de Buccinasco : dans ces différents cas, l'on peut en effet identifier des phases au cours desquelles l'administration a massivement tenté d'impliquer les parties dans le but de parvenir à une écoute réciproque maximale et à une bonne compréhension des points de vue respectifs de chacun, avec pour objectif de promouvoir un profond changement et d'aboutir à la réalisation d'une convergence certaine. L'on peut dans ce cas parler de médiation expressive (ou dialogique) : il ne s'agit en effet pas ici de faire d'une opposition un objet et, en conséquence, de tenter de parvenir à l'acceptation de normes communes. L'objectif est plus ambitieux. Il est de valoriser les capacités d'expression de chacune des parties. Il semblerait que ce type de médiation soit possible lorsque les institutions se fixent des objectifs d'intervention à large portée, mettant en place une stratégie de politiques intégrées et non spécialisées à l'attention des seuls Roms. Les politiques deviennent alors des facteurs de médiation expressive lorsqu'elles sont capables d'impliquer les parties dans la production de nouveaux symboles communs. Capables de mettre en discussion la mémoire collective, en incluant l'inscription des groupes tziganes dans l'histoire locale (About, 2015), en mettant en évidence des complémentarités possibles, mais aussi des signes d'appartenance commune. Les exemples typiques de ce genre de médiation sont fournis par des situations où un lieu est choisi pour l'implantation de logements à l'endroit d'un groupe tzigane et, en l'occurrence, lorsque ce lieu n'est pas occulté afin d'éviter des protestations. Mais quand il fait au contraire l'objet d'une publicité officielle, prévoyant des lieux dans lesquels discuter des problématiques et des hostilités soulevées par les habitants, créant ainsi les conditions de la confrontation et de la rencontre. Comme l'ont montré les cas de Settimo Torinese et de Buccinasco, la participation des habitants peut faire l'objet d'une médiation, directement et expressément, en discutant du projet d'implantation, et sur cette base, des problématiques du quartier de manière plus générale, le tout avec l'ensemble des parties en conflit.

\section{L'action collective des Roms comme levier de l'action publique locale}

L'évacuation sans alternatives et l'expulsion de ressortissants européens constituent des instruments à très bas dégrée d'efficacité, quelque soient les objectifs de politique publique (O'Nions, 2011). En France, Cousin et Legros ont bien montré que « les politiques répressives restent jusqu'à aujourd'hui un facteur secondaire dans les choix résidentiels des personnes expulsées » (2014, p.1282). Par contre, l'expression politique de la solidarité met en valeur des voies efficaces, durables, efficientes et capables de précisément redonner une perspective au vivre ensemble au sein de la cité. Et ceci vaut davantage encore lorsque les villes sont attentives aux mobilisations locales solidaires, et pas seulement aux mobilisations xénophobes et racistes. Lorsqu'elles se mettent en conséquence à penser des instruments d'action publique divers et multiples, et la médiation des conflits inévitables. Les Roms eux mêmes sont de plus en plus actif pas seulement avec des revendications de ressources mais aussi avec de demandes de reconnaissance. Pour beaucoup d'année la présence politique des activistes Roms, presque dans tout les Pays d'Europe (bien sur beaucoup plus dans les Pays de l'Europe de l'Est), a été influencé par le nationalisme, avec des revendications sur un registre nationaliste. Aujourd'hui par contre les Roms commence de plus en plus à développer des modalités de représentation situationniste, qui jouent sur les stéréotypes pour construire des évènements médiatiques $^{2}$. Plus en générale, plusieurs campagne d'opinion organisé par les associations Roms sont fondées sur un principe de lutte au « misérabilisme », sur une idée de inscription des Roms dans

\footnotetext{
${ }^{2}$ Pour un exemple à Rome : https://www.youtube.com/watch?v=i3OsW F7KHA, voir aussi à Paris https://twitter.com/Garriberts/status/453495806189109248/photo/1 . Les deux actions ont été faites le même jour, en avril 2014.
} 
des interactions ordinaires sur les lieux de travail, dans les marchés de la ville, dans les services publics et sociaux, dans l'imbrication des symboles qui forment la vie culturelle d'une ville ${ }^{3}$.

Les épreuves d'authenticité inventées par les Roms travaillent exactement sur l'expérience, et sur la narration et représentation de l'expérience pour agresser l'impossibilité du cosmopolitisme et le rendre possible.

Quelque exemple de ces formes d'action mérite d'être rapidement cité. Une forme d'action typique, très réfléchi et avec un haut dégrée de intentionnalité de familles roms qui vivent en bidonvilles consiste en inviter les riverains dans leur baraque pour montrer la qualité de l'espace habité (Bruneteaux, Benarrosh-Orsoni, 2012). Une deuxième forme d'action typique des familles roms consiste à envoyer leur enfants à faire du bénévolat dans les maisons de retraite (jouer avec les âgés, dessiner avec eux). Une troisième forme consiste à développer des stratégies de invisibilité très forte, à ne pas se déguiser en tsiganes, à cacher l'identité ethnique pour avoir des relations dans les espaces publics (c'est très diffusée entre les commerçants).

Bien sur, pas tous les roms sont prôneurs pour un cosmopolitisme stratégique des interactions et des liens sociaux; le repli communautaire est très fort et positivement corrélé à l'intensité des discriminations et de la ségrégation spatiale. Les réseaux complètement homogames existent, mais l'observation d'une variété plus large de forme d'action de Roms permet d'apprendre que il y a des délibérations intentionnelles et réflexives finalisées à créer des liens sociaux et des formes de reconnaissance ${ }^{4}$. Ces modestes formes de cosmopolitismes naissent hors des institutions, mais se renforcent quand s'appuient sur des collaborations avec des institutions (l'institution scolaire par exemple). Ces actions militantes ont un très bas dégrée de instrumentalité. Ne sont pas faites par faire des projets solidaires, ou obtenir réputation et financement. Bien sur, ils sont productif de capital sociale et byproduct, comme effets imprévus de programmes d'insertion professionnelle, pour l'emploi, orientation scolaire et professionnelle. Mais elles ne sont pas visées à ça. Elles demandent seulement d'être reconnues, pour ouvrir des opportunités d'apprentissage institutionnel pour les politiques sociales.

\section{Bibliographie}

About I. (2015), « Une nouvelle histoire des Tsiganes ? », La Vie des idées, 13 juillet 2015.

Aguilera T. (2014), «L'(in)action publique face aux squats discrets à Paris et à Madrid. Déni d'agenda et autonomisation de la sécurisation : comment la méconnaissance du territoire bloque les politiques publiques », Métropoles, $\mathrm{n}^{\circ} 14$, http://metropoles.revues.org/4860.

Aguilera T., Vitale T. (2015), « Les bidonvilles en Europe: une production politique. Controverses, action publique et effets», Revue Projet, à paraître.

(van) Baar H. (2011), « Europe's Romaphobia: problematization, securitization, nomadization », Environment and Planning D: Society and Space, no. 29 (2), p. 203-12.

Belqasmi M. (2014), « La construction d'une "question tsigane" : entre catégorisations et mobilisations sociales », Migrations Société, no. 152, p. 49-58.

Berger P.L. (sous la direction de) (1998), The Limits of Social Cohesion, Boulder-Oxford, Westview Press.

Bessone M., Doytcheva M., Duez J.-B., Girard C., De Latour S. G. (2013), « Integrating Or Segregating Roma Migrants In France In The Name Of Respect: A Spatial Analysis Of The Villages D'insertion », Journal of Urban Affairs, no. 36, p. 182-196.

Bruggeman D. (2011), "Conditions d'enquête et démarches méthodologiques de recherches «à domicile ». Le chercheur sur le terrain des familles », Les Sciences de l'éducation - Pour l'Ére nouvelle, no. 44 (4), p. 51-73.

Brüggemann C. (2012), Roma Education in Comparative Perspective. Analysis of the UNDP/World

\footnotetext{
${ }^{3}$ https://www.youtube.com/watch?v=JKvcApXoTRg\&index=6\&list=PLitMY-Sk7Sb-OzgXbMu4gomQyqzMcxMxk.

4 Une exemple emblematique est la campagne italienne «Rom citoyens de l'Italie en train de se faire »: https://www.youtube.com/playlist?list=PLitMY-Sk7Sb-OzgXbMu4gomQyqzMcxMxk.
} 
Bank/EC Regional Roma Survey 2011. Roma Inclusion Working Papers, Bratislava, United Nations Development Programme.

Bruneteaux P., Benarrosh-Orsoni N. (2012), Intégrer les Rroms ?, Paris, L'Harmattan.

Cousin G. (2013), "L'évacuation de bidonvilles roms. Circulaires et cycles médiatiques », Métropolitiques, 18 novembre 2013. URL : http://www.metropolitiques.eu/L-evacuation-debidonvilles-roms.html.

Cousin G., Legros O. (2014), « Gouverner par l'évacuation ? L'exemple des campements illicites en Seine-Saint-Denis », Annales de Géographie, 700, p. 1262-1284.

Doytcheva M. (2015), « Roms et Tsiganes en Europe méditerranéenne : l'actualité d'une question », Confluences Méditerranée, no. 93, p. 9-25.

Duvoux N. (2012), Le Nouvel Age de la solidarité. Paris, La République des idées.

Halpern C., Lascumes P., Le Galès P. (sous la direction de) (2014), L'instrumentation de l'action publique, Paris, Presses de Sciences Po.

Hirschman A.O. (1991), Deux siècles de réthorique réactionnaire, Paris, Fayard, 1991.

Legros O. (2010), «Les pouvoirs publics et les grands « bidonvilles roms » au nord de Paris (Aubervilliers, Saint-Denis, Saint-Ouen)», http://espacestemps.net/document8422.html.

Legros O., Olivera M. (2014), « La gouvernance métropolitaine à l'épreuve de la mobilité contrainte des Roms migrants », EspacesTemps.net, Travaux, 21.03.2014.

Legros O., Vitale T. (2011), « Les migrants roms dans les villes françaises et italiennes : mobilités, régulations et marginalités », Géocarrefour, no. 86 (1), p. 3-14.

Mantovan C. (2012), "Citoyens ou étrangers ? " Dynamiques d'inclusion et d'exclusion dans le conflit du village Sinti de Venise, sEepiDéviance et Société, no. 36 (1), p. 37-60.

Mayer N., Michelat G., Tiberj V., Vitale T. (2014), " La persistance des préjugés anti-Roms », in Commission nationale consultative des droits de l'homme, La lutte contre le racisme, l'antisémitisme et la xénophobie. Année 2014, Paris, la Documentation Française, pp. 251-260.

Naçu A. (2010), «Les Roms migrants en région parisienne : les dispositifs d'une marginalisation », Revue européenne des migrations internationales, no. 26 (1), p. 141-160.

O’Nions H. (2011), « Roma Expulsions and Discrimination: The Elephant in Brussels », European Journal of Migration and Law, no. 13 (4), p. 361-388.

Olivera M. (2014), « Le Hanul de Saint-Denis. Du bidonville au "campement illicite". La question rom en France et en Europe », in Agier M., Lecadet C. (sous la direction de), Un paysage global des camps, Paris, La Découverte.

Olivera M. (2015), « Insupportables pollueurs ou recycleurs de génie ? Quelques réflexions sur les «Roms » et les paradoxes de l'urbanité libérale », Ethnologie française, vol. XLV, no. 3, p. 50313.

Sarcinelli A. S. (2012), « "Des gamins en colère, perdus, sans espoir”. L'enfance rom en Italie entre condamnation et compassion ", in Fassin D., Eideliman J. (sous la direction de), Economies morales contemporaines, Paris, La découverte, p. 193-211.

Simmel G. (1908, tr. 2003), Le conflit, Strasbourg, Circé, 2003.

Stewart M. (2013), " Roma and Gypsy "Ethnicity" as a Subject of Anthropological Inquiry », Annual Review of Anthropology, no. 42 (1), p. 415-432.

Vitale T. (2009), « Politique des évictions. Une approche pragmatique », in Cantelli F., Roca i Escoda M., Stavo-Debauge J., Pattaroni L. (sous la direction de), Sensibilités pragmatiques. Enquêter sur l'action publique, Bruxelles, P.I.E. Peter Lang, p. 71-92.

Vitale T. (2010), «Le basi cognitive degli interventi educativi con i sinti. Eredità, continuità, stratificazioni e cambiamenti », Animazione Sociale, no. 241, p. 34-43.

Vitale T. (2015a), «Les politiques locales face aux Roms : entre réification, effets de visibilité et reconnaissance », Métropolitiques, 4 February 2015. URL: http://www.metropolitiques.eu/Lespolitiques-locales-face-aux.html.

Vitale T. (2015b), «Rom a Roma: è arrivato il momento di giocarsi il sette bello. 7 principi di 
programmazione per implementare una politica efficace di superamento dei campi nomadi » in Associazione 21 luglio, I centri di raccolta spa. I centri di assistenza abitativa per soli rom. I costi a roma nel 2014, Roma, pp. 57-71.

Vitale T., Boschetti L. (2011), " "Les Roms ne sont pas encore prêts à se représenter eux-mêmes !" Asymétries et tensions entre groupes Roms et associations 'gadjé' à Milan », in Berger M., Cefaï D., Gayet-Viaud C. (sous la direction de), Du civil au politique. Ethnographies du vivreensemble, Bruxelles, P.I.E. Peter Lang, p. 403-29.

Vitale T., Caruso L. (2009), «Ragionare per casi: dinamiche di innovazione nelle politiche locali con i rom e i sinti », in Vitale T. (sous la direction de), Politiche possibili. Abitare le città con i rom e i sinti, Roma, Carocci, p. 265-88.

Vitale T., Claps E., Arrigoni P. (2009), « Regards croisés. Antitsiganisme et possibilité du vivre ensemble, Roms et gadjés, en Italie », Etudes Tsiganes, no. 35, p. 80-103.

Vitale T., Cousin B. (2011), «En Italie. Scolarisation des Roms et des Sintis », Cahiers pédagogiques, HSN no. 21, p. 164-6.

Vitale T., Membretti A. (2013), « Just another roll of the dice: A socially creative initiative to assure Roma housing in North Western Italy », dans F. Moulaert, D. MacCallum, A. Mehmood et A. Hamdouch (eds), International Handbook on Social Innovation. Collective Action, Social Learning and Transdisciplinary Research, Cheltenham: Edward Elgar, p. 186-196. 